\title{
Possibilities and constraints in the use of very high spatial resolution UltraCamX airborne imagery and Digital Surface Models for classification in densely built-up areas - a case study of Berlin
}

\author{
Corinna Brüßhaber*a , Anna Maria Trosset ${ }^{\mathrm{a}}$, Tilman Bucher ${ }^{\mathrm{a}}$ \\ ${ }^{a}$ German Aerospace Center (DLR e.V.), Institute of Robotics and Mechatronics, \\ Dept. of Applications and Sensor Concepts, Rutherforstr. 2, 12489 Berlin
}

\begin{abstract}
With the availability of large digital frame cameras like the UltraCamX (UCX) additional benefits through a combination of high-resolution multispectral aerial images with highly accurate digital surface models emerge. This ongoing study examines the level of detail of urban information that can be extracted. High resolution and the unprecedented geometric accuracy of the multispectral and the 2.5D object information enable the derivation of detailed and characteristic object features. The method of object-based classification is not only used to extract meaningful objects, even more important is a detailed assessment of semantic relationships. Our study shows the explicit advantage of high geometric resolution to increase the stability of classification and the number of classes in a representative area of Berlin.
\end{abstract}

Keywords: UltraCamX, very high resolution, DSM, true orthoimage, urban remote sensing, soil sealing, urban structure, object-oriented classification, Berlin

\section{INTRODUCTION}

The German Aerospace Centre (DLR) in Berlin-Adlershof covers the complete chain of image generation and processing, starting with sensor design and construction, image acquisition, processing and interpretation. The DLR has to its disposal high-resolution multitemporal and multisensoral data sets including true orthoimage mosaics (TOM) and digital surface models (DSM) of the whole city area of Berlin. To obtain detailed and very precise thematic and geometric information on urban objects - such as built-up areas with detailed housing characteristics, statements concerning the nature and degree of soil sealing, extend and spatial distribution of land cover and urban structure object-oriented methodologies are used to get comprehensive object features.

This paper is the first step of an ongoing study which is conducted in the context of two current PhD theses at the DLR in Berlin-Adlershof. One goal of this introductory step is to present the novel multispectral and height information data sets of Berlin which were acquired by the matrix camera UltraCamX (UCX) and processed with the automatic processing chain at the DLR. Furthermore we present additional benefits of this kind of data to issues of urban remote sensing. This is done by pointing out the urban land cover and urban structure in a representative part near the city centre of Berlin. It is performed with suitable methodology like object-oriented classification and spatial analysis in Geographical Information Systems (GIS). Difficulties in the classification within shadows, especially the problem of not visible areas within inner courtyards as typical phenomenon of Berlin tenements are analyzed; they will be addressed in subsequent studies. It is planned to transfer the developed rule base and the gained knowledge to the whole city area of Berlin.

*corinna.bruesshaber@dlr.de; phone +49 30 67055-367; fax: +49 30 67055-385; www.dlr.de/os

Earth Resources and Environmental Remote Sensing/GIS Applications, edited by Ulrich Michel, Daniel L. Civco, Proc. of SPIE Vol. 7831, 783115 · @ 2010 SPIE · CCC code: 0277-786X/10/\$18 · doi: 10.1117/12.865826 


\section{GEOGRAPHIC BACKGROUND}

Airborne remote sensing is a valuable instrument for urban applications. Very high resolution aerial images combined with the corresponding digital surface models (DSM) are useful for diverse questions in urban ecology and urban planning; they are employed to detect more detailed surface features on object level. Very large scales (1:1000 - 1:5000 and lower) [2] can be used. Previous studies usually remain on the house or block level [3]. Highly accurate modelling of buildings allows reliable measurements of e.g. air flows which are changed by buildings.

Because of the extensive historical background, Berlin has various faces. This fact results in very dense built-up areas close to the city centre and in the neighbourhood of spacious developments, lots of green and open spaces in more remote areas. Beyond that the region Berlin has much more vegetation, lakes and rivers than many other cities in Germany.

Municipal sealing and urban structure is of interest to many environmental and social issues. Suburbanization in the outskirts of Berlin with expansion of sealing is one related process to human settlement which needs continuous monitoring. Urban settlements and developments change the cityscape and cause climate changes [4]. A continuous recording and monitoring of these corresponding indicators is therefore significant. City ecologists evaluate sealing data, solutions are developed which determine the necessary action. In focus of interest especially for public utilities and solar energy providers is also the detailed extraction of roof surfaces and roof constructions (e. g. dormers and chimneys), which was investigated in previous studies at the DLR based on an UltraCamD data set [5]. Very high resolution surface models are indispensable for such tasks.

\subsection{Soil sealing}

One of the most specific frequent changes of urban area results from the soil sealing. A previous study investigates the urban sealing of Berlin based on satellite images combined with data of the Berlin Digital Environmental Atlas, sealing data determined by the Berliner Wasserbetriebe and conventional aerial images [6].

The degree of soil sealing is an important parameter that has to be monitored as it has a noticeable influence e. g. on the urban climate. It is one measurable quantity in the urban context which can be calculated on basis of remote sensing techniques. The average degree of sealing is composed of the proportion of the built-up and other sealed area in relation to the total area of the block. Spectral features, texture and elevation information and a geometric component are used to separate the different types of land cover, the geometric / height component also supports the spatial dimension (elevated or non-elevated). The main cause of urban climate change is the conversion of natural land in urban sealed surfaces, which mainly consist of synthetic materials. This implicates a change in the biosphere by reduction of vegetation covered surface; the degree of vegetation is one of the urban structure dimensions. Other typical urbanization phenomenons are an increase of roughness and sustainable changes of physical surface properties like reflectance (urban albedo), absorbance and heat capacity. Another consequence is the poor irrigation of green space and urban vegetation in general. Rain water flows off too fast, therefore insufficient amounts of water evaporates into the city air which is finally stored in the soil [7]. Furthermore the urban space is heating up.

Around the historical centre of Berlin the built-up structure consists of old buildings, especially tenements. Typical tenements were built mainly in the first part of the last century during the industrialization. Tenements are inner-city multi-storey residential complexes with one or more inner courtyards. These inner courtyards are one of the biggest problems in the question of degree of urban soil sealing. It is of interest to city planner and political decision makers, building owners and utilities. Therefore, it is an attractive topic for the processing of remote sensing data and one specific part of this study. Due to their geometric properties the use of an orthogonal projected image (True Orthoimage Mosaic - TOM) and a DSM is essential, which is a prerequisite to avoid artefacts like perspective displacements and areas occluded by elevated objects/buildings. Moreover buildings are not distorted from their true location. [8] The geometric accuracy of true orthoimages is much higher compared to conventional orthoimages. Due to large view angles and low overlap in many multispectral remote sensing products an inadequate visibility in a lot of courtyards is given. Furthermore the height of buildings and the non-ideal position of sun during the acquisition of aerial images results in shaded surfaces, especially in small inner courtyards. Often the radiometric resolution is insufficient for classification. Another problem in this context arises in presence of trees, especially during flight campaigns in the summer months. The view on the bottom of the courtyard is for the most parts prevented by leaves. The proportion of sealed areas and additional the used materials can be recorded detailed based on very high resolution image data. One advantage of digital 
camera systems is the high radiometric and geometric resolution [9], [16]. Very small surface structures on very heterogeneous surfaces - a typically characteristic for urban areas - can detected and moreover segment based extracted.

\subsection{Urban structure}

Structure types are integrated as a basic dimension in the Berlin Digital Environmental Atlas [18]. Sections are divided into characteristic structure types based on the actual use and their related appearance. They were defined in the official Land Use Plan of the Senate Department of Urban Development previously. The urban planner fundamentally distinguishes between sections of predominantly residential use, predominantly commercial, service, small business and industrial areas and other sections like public facilities, traffic areas, construction sites or green and open spaces [10], [19]. All these areas are covered by statistical blocks and differentiate different units of use. Based on this knowledge it is possible to derive the degree of soil sealing, the degree of vegetation density and also population densities and further social issues with methods of very high resolution remote sensing. Especially for sparsely mapped towns in less developed regions on earth this is of great importance. Using a combination of different scales within one project a more meaningful analysis of semantic relationships is carried out.

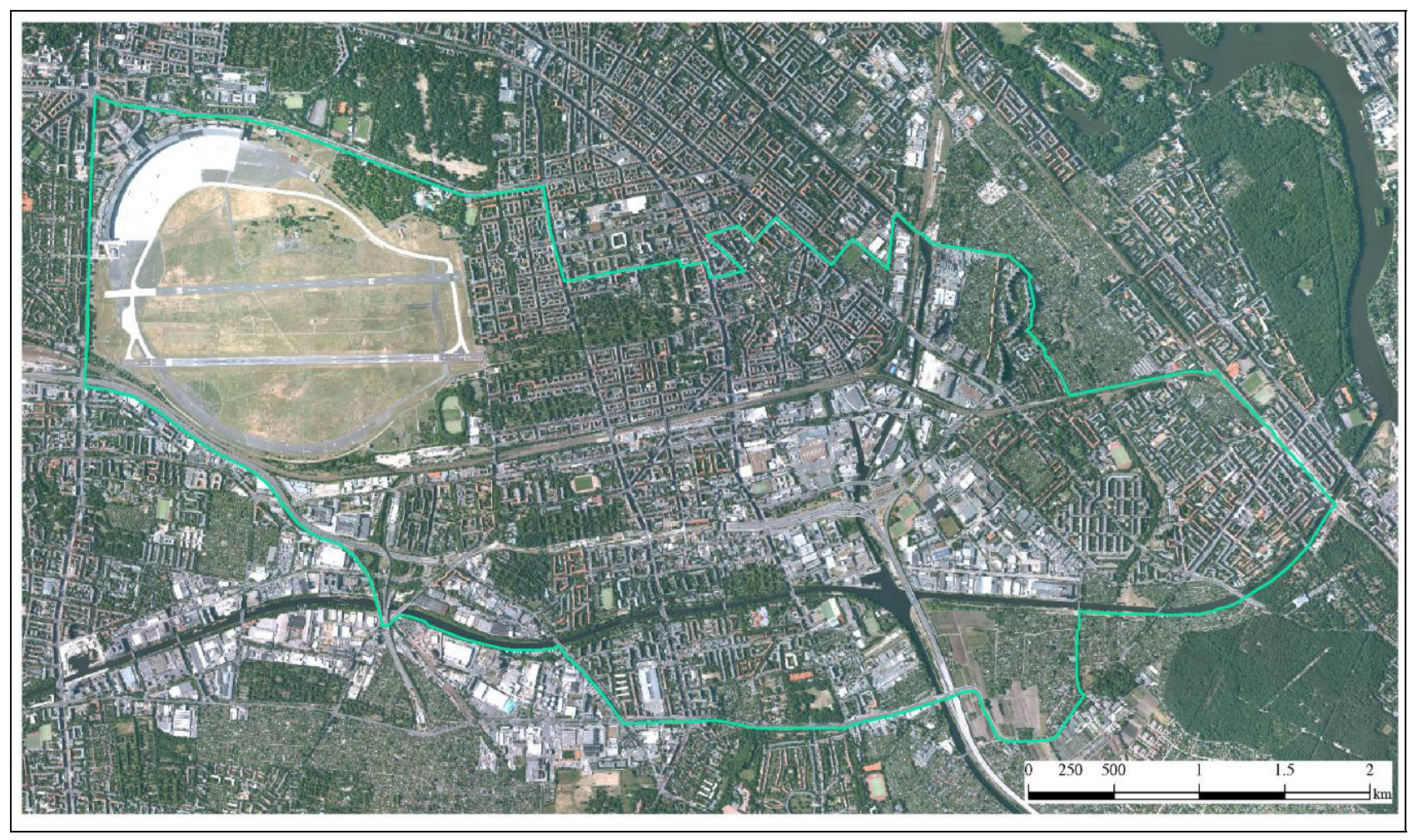

Figure 1: Study area in the south of Berlin (Berlin Tempelhof Airport) - UltraCamX true orthoimage (original resolution $15 \mathrm{~cm} / \mathrm{pixel})$.

\section{STUDY AREA AND DATA SETS}

The selected study area is situated in the city centre of Berlin (see figure 1) close to the historical almost hundred years old Berlin Tempelhof Airport, who has been out of service since the end of 2008. Following the closure of the traditional city airport, the area has been characterized by a high conversion rate. The study area is a non-rectangular subset with an extent of $45.000 * 25.000$ pixels in the southern inner-city of Berlin. It covers an area of $16 \mathrm{~km}^{2}$ and includes typical urban land cover and structure types. It is characterized by a variety of different classes such as sections with residential use (tenements, allotments), traffic areas (railway, city highways and the former airport), industry and wasteland, 
waterways, green and open space (parks) as well as by a high heterogeneity within the object categories. Parts of the Barnimer glacial valley are included in the study area, the local elevation difference amounts up to $15 \mathrm{~m}$.

\subsection{Image data and derived products}

The UCX is a large format digital aerial frame camera with panchromatic and additional multispectral capabilities. Typical image resolution which are used at the DLR in Berlin for urban applications range between 7 and $20 \mathrm{~cm}$. Using the SGM, a complex matching algorithm which was developed at the DLR [1], a detailed digital surface models (DSM) in full image resolution is generated. Large true orthoimage mosaics (TOM) in 4 multispectral channels (infrared, red, green and blue) of extensive areas are derived. Both, DSM and TOM perfectly fit to each other and display very sharp object contours; they are used for this study. The geometric resolution of the used image data is $0.15 \mathrm{~m}(\mathrm{x} / \mathrm{y})$, a standard radiometric depth of 8 bit / channel was used for the classification. Previous studies for other towns were carried out using the full radiometric resolution (16 bit data format); which is not a standard procedure. For the 2008 Berlin data 16 bit data sets will be compared to the 8 bit data in the near future and promising results are expected. A more detailed description of the DSM / TOM data processing is given in [9], [17].

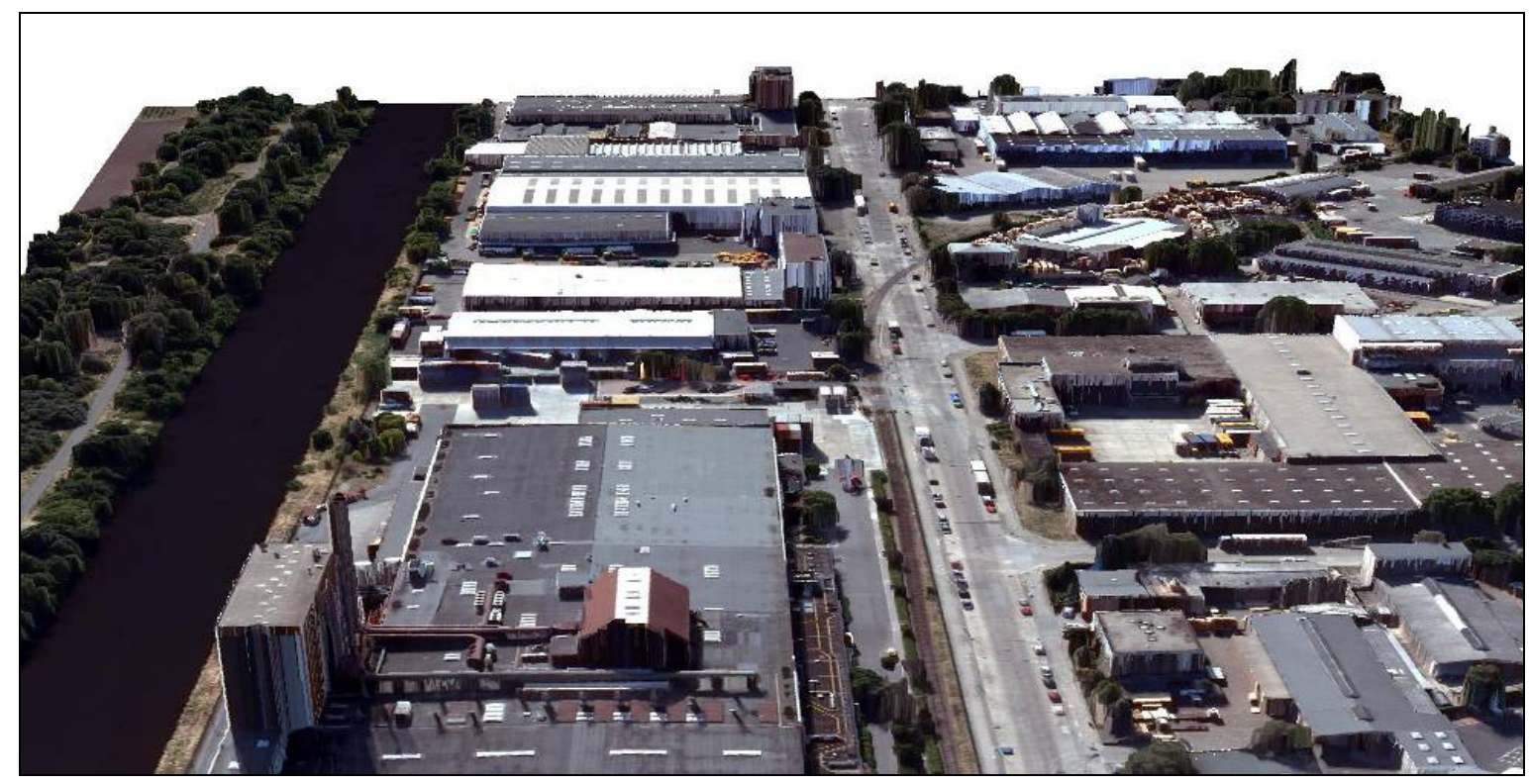

Figure 2: 3D-surface view of the DSM automatically textured with the true-orthoimage; structure type: predominantly commercial, service and industry with green spaces and water body.

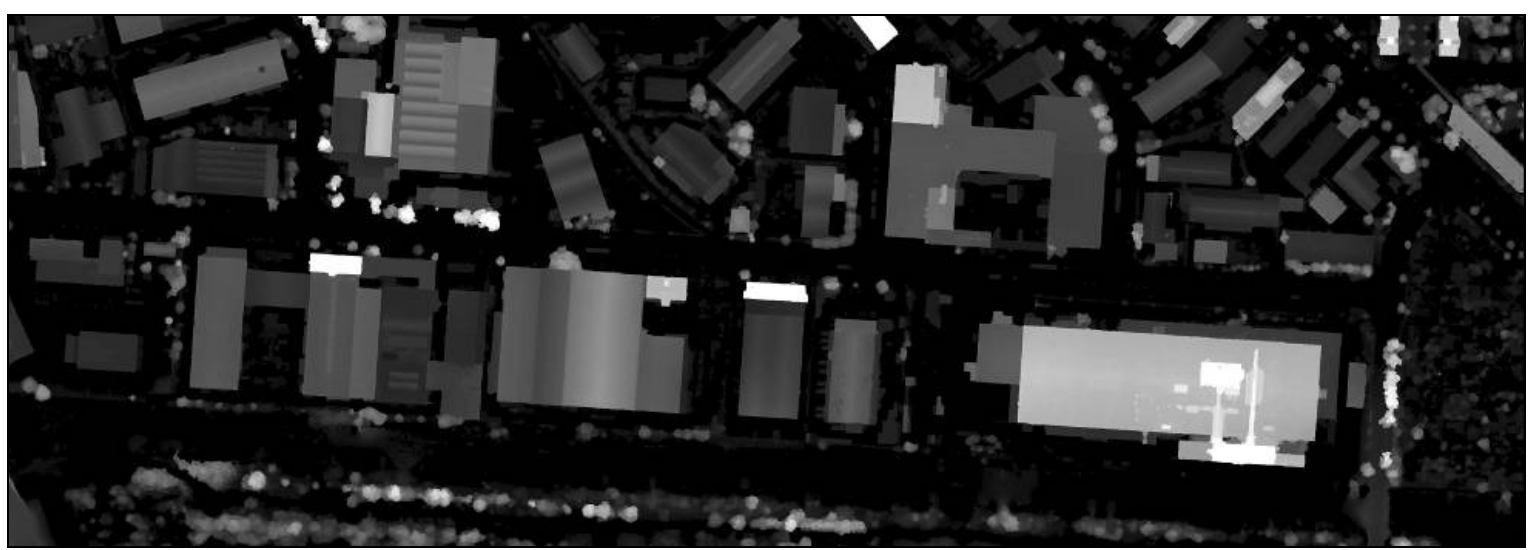

Figure 3: color coded DSM (90 rotated). 
Additionally a normalized DSM (nDSM) is calculated to determine the absolute object heights.

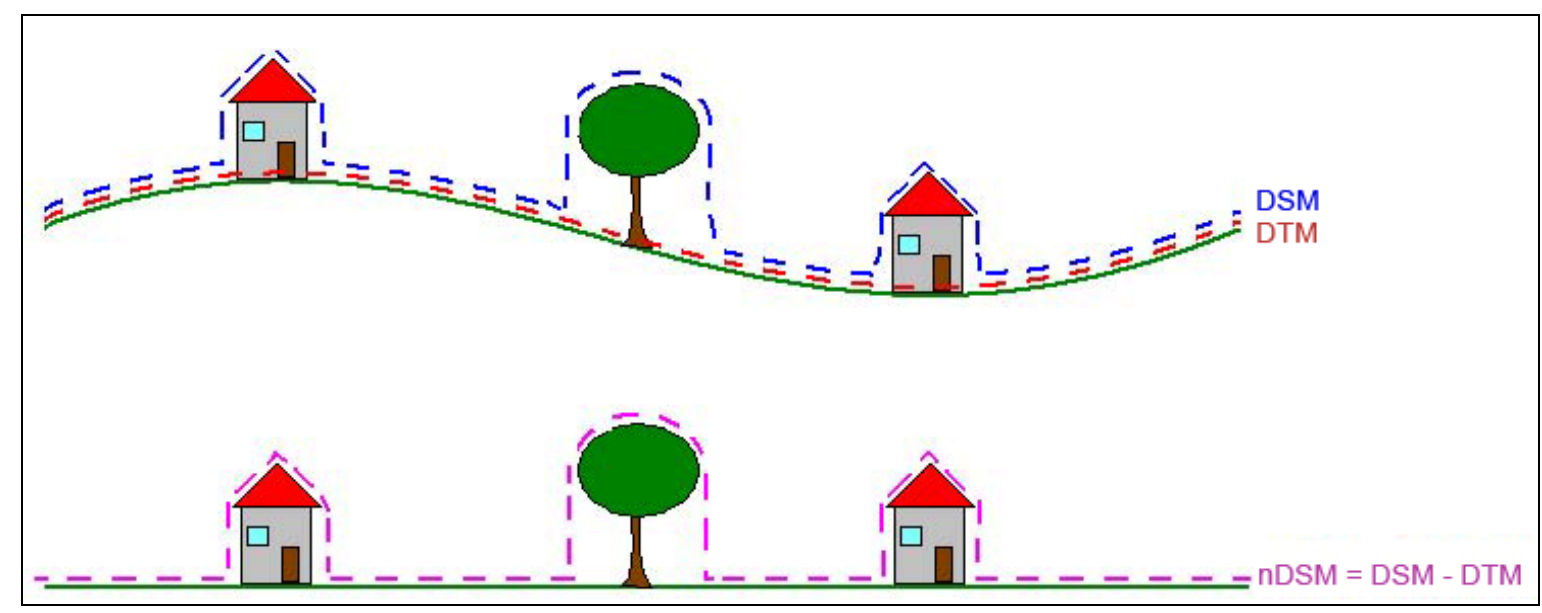

Figure 4: DSM, DTM and nDSM.

A normalized Digital Surface Model (nDSM) is the difference between the surface model (DSM) and terrain model (DTM). The nDSM shows only the buildings and vegetation that stand out from the site (terrain), all other surfaces have a constant height zero (see figure 4). In this way simple classification rule sets can be used to distinguish between ground and non-ground. An almost automatic procedure was used, which was developed at DLR by [11]. The DTM is searched in this algorithm step for edges and abrupt change of altitude. Points are identified which are located directly and with high probability at the ground terrain. When sufficient ground points are known, they are interpolated to a closed surface and all non-ground objects can automatically be removed. The resulting DTM allows then, by subtracting the DTM from the DSM, the calculation of the nDSM.

In addition to the multispectral true orthoimage, the DSM and the nDSM two official data sets of the Berlin Senate, the Automated Cadastral Map (ALK) from the year 2006 and the statistical block map from 2008 are used for the classification procedure.

\subsection{Automated Cadastral Map and Statistical Block Map}

The automated cadastral map (ALK) is an official part of the land register. It stores the geometric information as well as the appropriate factual data - the number of storeys and the type of building. The ALK serves as a reference to the detected building geometries in the aerial images. The ALK contains the entire estate of Berlin as closed polygon coverage. The polygons of the automated cadastral map are covered by the limits of the statistical block map (1:5000).

Another official reference is the statistical block map (ISU5) of the Senate Department for urban Development Berlin (Dec/2008). It is a vector map (1:5000) and shows each individual statistical block, which is delimited by streets. It contains the land use, the urban structural type and the area type of block and block segments. Numbering and limitation of the blocks are carried by the State Statistical Office.

\section{METHODOLOGY}

Two independent analyses were applied to the available data set of UCX in the chosen study area in Berlin. On the one hand an object-based classification was used in order to evaluate the suitability of this method for semi-automatic extraction of relevant features for urban planning and to show the advantages of high resolution and position accuracy of the UCX data for classification purposes. On the other hand the classification results were implemented to a GIS and intersected with the official statistical block data due to analyse the common urban items, like the degree of soil sealing or proportions of green and open spaces. Furthermore the constraints of the approach should be investigated due to limitations of large data handling. As mentioned above the data processing chain of the DLR was used to generate most 
of the input data for the interpretation. The segmentation and classification processes are performed in the commercial software Trimble eCognition in version 8.0. The method of object-based classification is not only used to extract meaningful objects, but also neighbourhood relationships and relevant object attributes due to extract semantic context and interrelation between the proportion of different features within statistical blocks. Only in this way a determination of urban structures is significant. The extraction of detailed surface characteristics and attributes like absolute height based on nDSM or texture metrics for surface cover are crucial for estimation of proper degree of soil sealing, also partially in shadowed parts of image. The classification of shaded areas shows already on the basis of 8 bit data good results; the reliability will be improved in the near future due to the 16 bit data which is being processed now.

A demanding task for the future is to additionally analyse specific composition and configuration of extracted features within blocks, like proposed by [12]. Moreover the implementation of the third dimension to describe urban metrics is another challenging task for the subsequent studies [15].

\subsection{Segmentation and classification}

The research pursues an approach for the extraction of urban features in two stages. Primary typical coarse urban features like buildings, roads, sealed surfaces and unsealed surfaces, vegetation, rails and water bodies are extracted. In the next step resulting relevant classes are the subject to a more detailed analysis. This considers in particular sealed surface habits (distinguishing between coverage materials due to its water permeability, e.g. large concrete slabs with thin gaps or cobblestones with large unsealed spaces or sandy surfaces) as well as the extraction of single roof tiles, which was already investigated in the previous study [5]. Such detailed analyses are only possible on the basis of the available very high geometric and radiometric resolutions.

Both stages are applied on single scene subsections, which represent a merge of several neighbouring statistical blocks with similar urban structures. The segmentation and object-based classification are implemented successively on every subsection. The results which are achieved on these single parts are then synchronized one after the other into the whole scene. This procedure speeds up the analysis, due to the fact, that the rule set is developed and optimized at only one small representative subset; it can be easily transferred to the whole scene using the batch processes and server technology. Owing to the fact, that the subsections are delineated along homogenous features like streets or water bodies, there are no problems with the artefacts at the subsection borders.

To sum up the analysis include following main steps: (1) generation of nDSM and additional layers in image processing software, (2) tiling of the scene in subsections, (3) initial segmentation (4) object-based classification interleaved with further segmentations, (4) synchronization of the classification results of the particular subsections to the whole scene, (5) accuracy assessment and in a final step (6) export and integration of the results in a GIS. All these steps are saved in a hierarchical process tree and can be easily edited and adapted in the case of errors as well as applied to other data sets.

Both multispectral and nDSM data as well as additional layers, like edge detection or aspect layer, were used in the segmentation and classification process. For the creation of segments, which should be a stable basis for the subsequent classification, the multiresolution and chessboard segmentations as well as the pixel based region growing and shrinking algorithms were used. The resulting objects, which are crucial for the success of the subsequent classification, represent meaningful semantic objects of the real world structures [13]. In this way the object-based classification approach comes closer to the real world characteristics than the pixel-based [14].

Owing to a very high spatial resolution of the UCX data the spectral heterogeneity of the objects emphasizes the complexity of the automated extraction of the urban features. Due to the fact that the rule base should be transferred to different in kind subsections it is composed of very robust class descriptions, at best of variables for summary statistics (e.g. quartiles are computed and stored as a variable). Feature variables allow developing rule sets in a more abstract form, as they are independent of specific feature types and data characteristics. However it is not always avoidable to use hard thresholds.

The crucial advantage of object-based classification methods is the possibility to use characteristics like shape, textures and neighbourhood relationships in addition to spectral features. This is essential for the successes of the detailed classification because particular class descriptions can be formulated very accurately so that even similar classes can be separated. All features can be exported to the attribute table and be used in further GIS-analyses.

For the extraction of buildings the ALK was used, since it represents the base lines which generally can not be extracted from aerial images due to roof projections. Moreover in the most cases ALK corresponds to the true soil sealing by buildings. In order to avoid errors caused by new buildings which are not contained in the available ALK, but also errors 
caused by false polygons (underground basements, too small objects) these building objects were extended. For this purpose pixel based growing algorithms were used. To restrict the growing process, conditions for features like absolute height and aspect as well as a fixed threshold for the edge detection layer were set. Pixels corresponding to the edges are not contained in the resulting building objects, they remain unclassified.

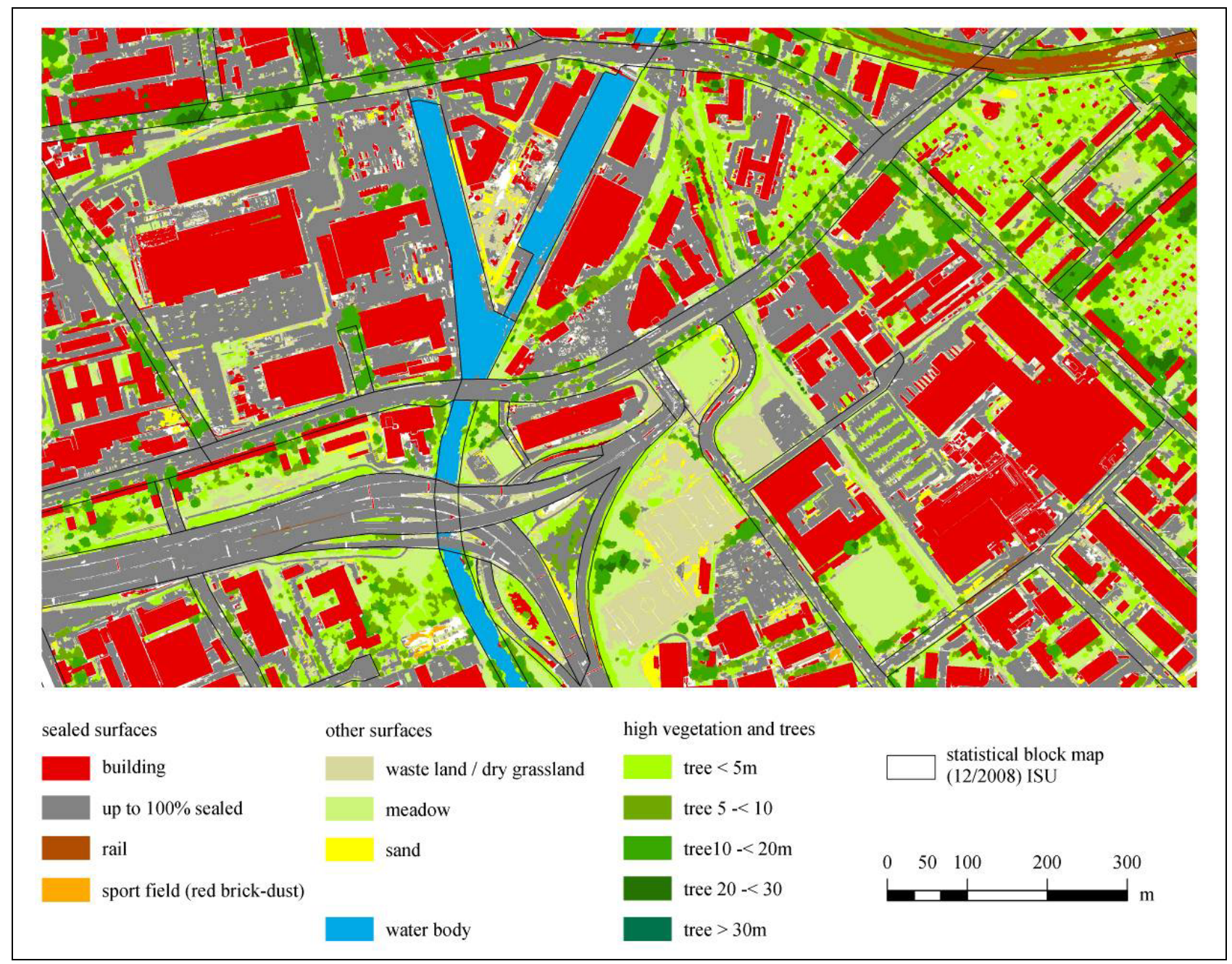

Figure 5: Classification result for a section of the entire study area overlaid with the statistical block map (1:5000).

For the classification of vegetation the NDVI (normalized differential vegetation index) and a ratio infrared / green channel were used. The combination of both shows very stable results also in shadowed areas and inner court yards. For the extraction of other features mostly the statistics for multispectral data but also shape and texture characteristics were used. In this study also the graphical user interface (the so called solution with libraries) was developed for some processes. This was necessary to allow the user influence over the estimation of parameters which have to be adapted to a different subsection characteristic. For example it is not reasonable to use the same segmentation parameters for a subsection with industrial character as for the residential one. At this stage the intervention of the user is required. 


\section{RESULTS AND DISCUSSION}

\subsection{Classification results}

The classification results were exported as vector and implemented into the GIS (see figure 5). At this stage the possibilities of the available data set for urban planning purposes can be shown. Due to a high extraction accuracy in conjunction with a very accurate nDSM not only the degree of soil sealing and proportions of different surfaces per block are estimated but also other urban items, like density of built-up area or the floor area ratio (FAR). The last one can be even carried out for single houses (house level) and not on the block level due to application of procedures which allow the extraction of single roofs and roof tiles.

Due to the fact that this study is still ongoing only an exemplary analysis of surface proportions per block is presented in this section. After a more detailed classification of sealed and unsealed areas, which is planned as a next stage, different weighting factors for various cover materials can be calculated, so that we can determine a proper degree of soil sealing.

Table 1: Surface proportions per block [\%].

\begin{tabular}{|l|c|c|c|c|c|}
\hline & Building & Sealed surface & Unsealed surface & Vegetation > 2m & Unclassified \\
\hline (1) Dense built-up & 64,39 & 2,02 & 10,54 & 20,08 & 2,97 \\
\hline (2) Loose built-up & 16,52 & 7,52 & 35,39 & 38,79 & 1,78 \\
\hline (3) Industry & 49,52 & 14,53 & 19,80 & 8,82 & 7,33 \\
\hline
\end{tabular}

Within these blocks the percentage of built-up, sealed and unsealed areas as well as proportion of vegetation were calculated (see table 1). Three exemplary blocks with different characteristics were chosen: (1) residential dense built-up area, (2) residential loose built-up area and (3) industrial area (see following figures, legend see figure 9 and 12).

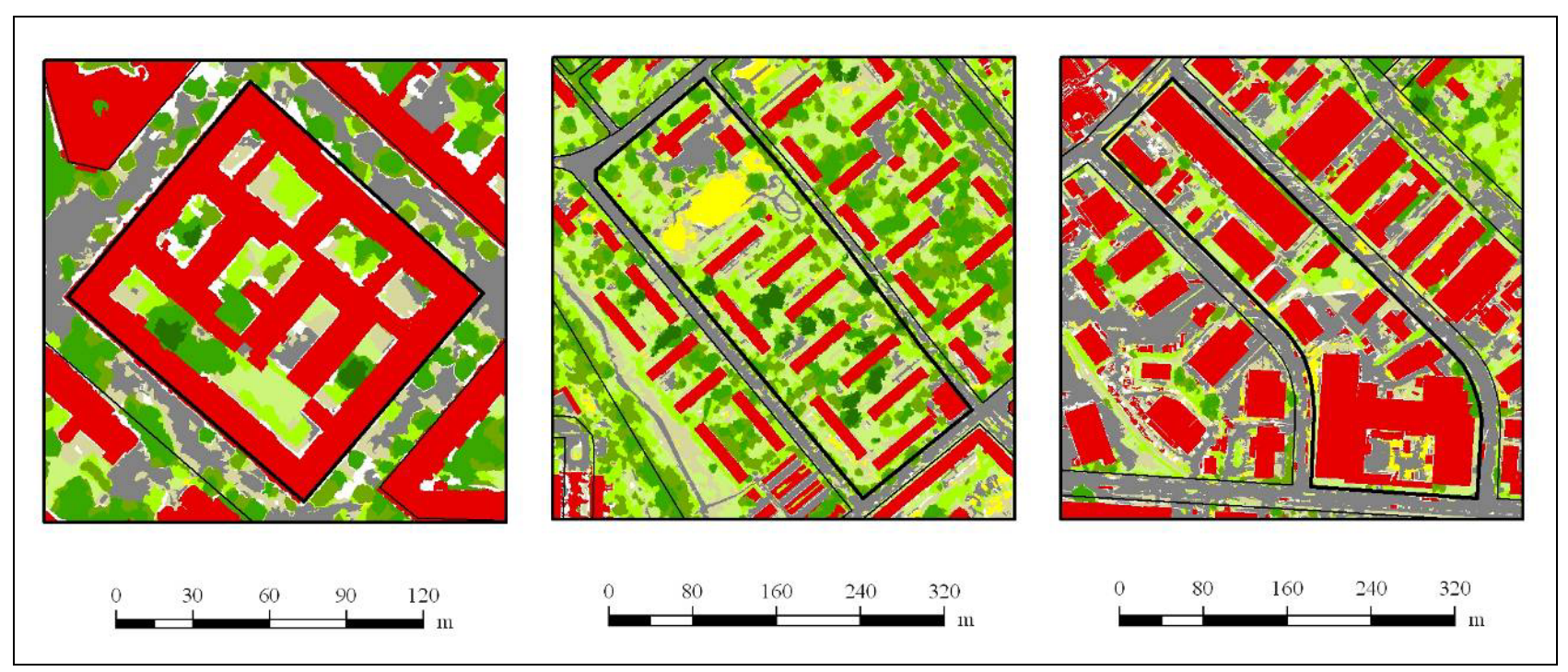

Figure 6: Example calculated on the block level: (1) dense built-up (2) loose built-up and 3) industrial area. 


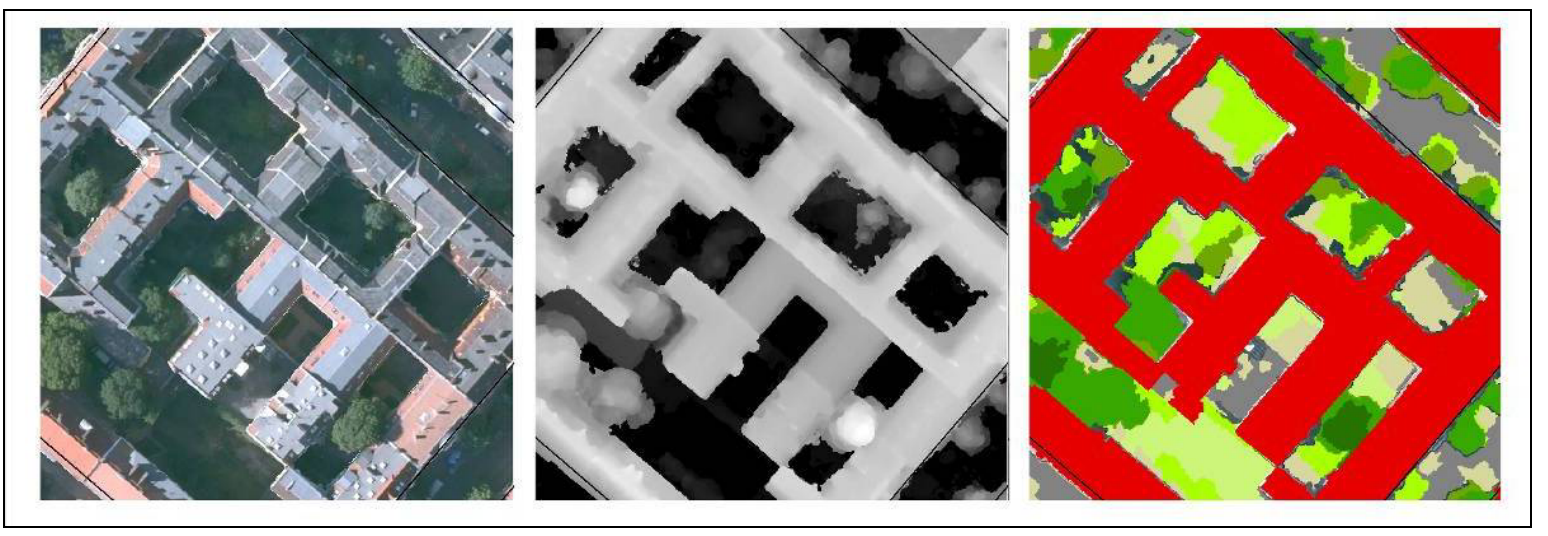

Figure 7: Classification result in structure type predominantly residential use (typical tenements); left: orthoimage; middle: DSM; right: classification result.

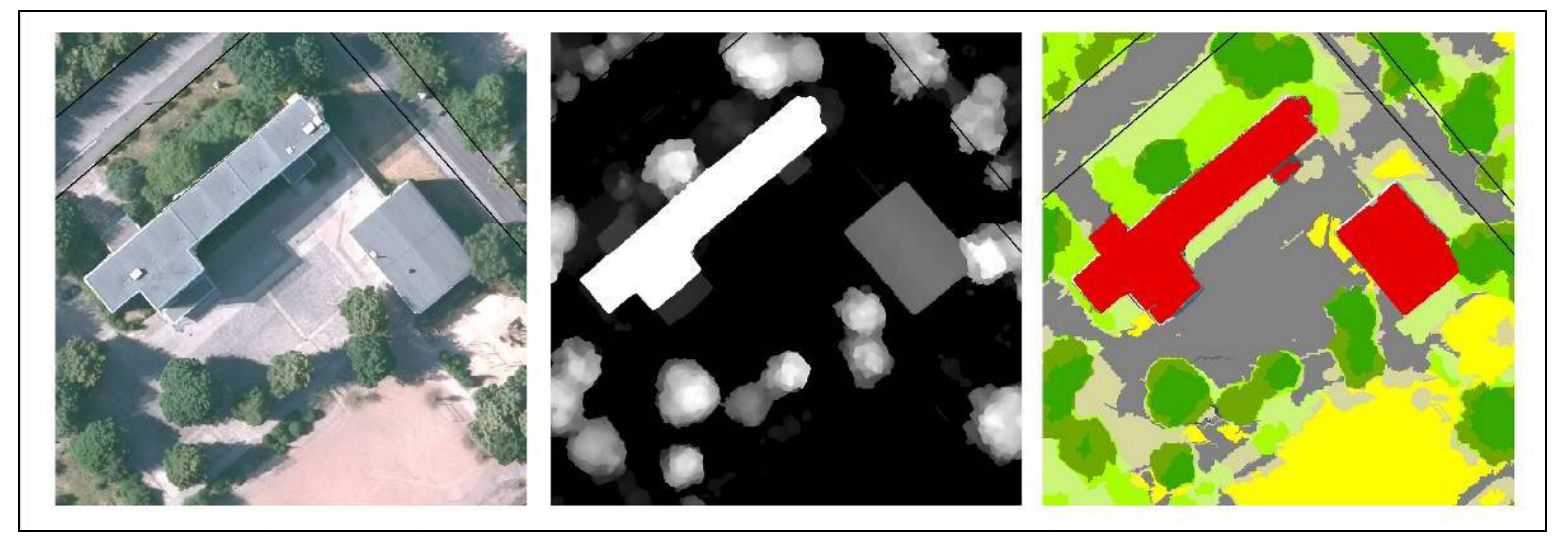

Figure 8: Classification result around a school building with sections of sand.

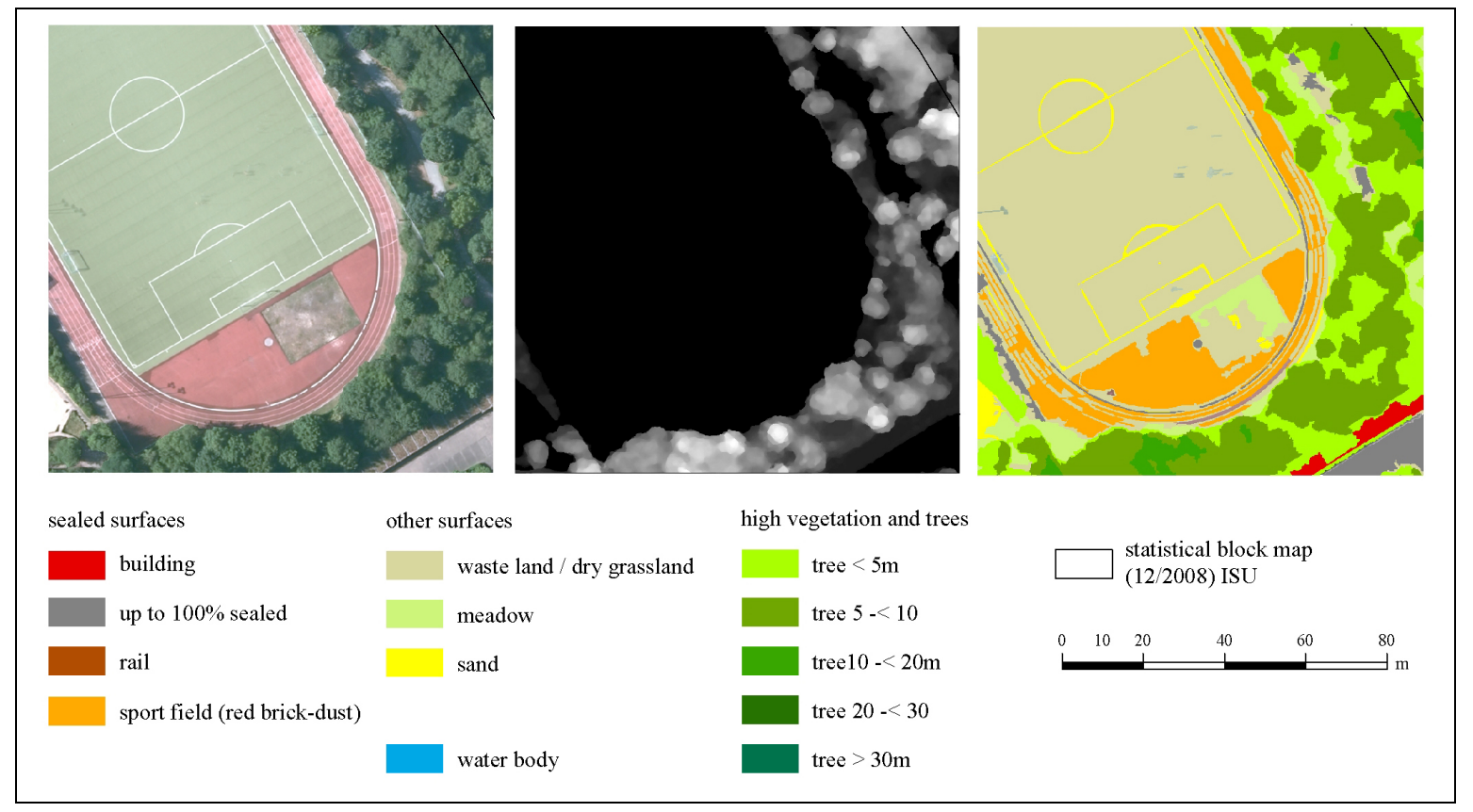

Figure 9: Classification result for a typical sports ground. 


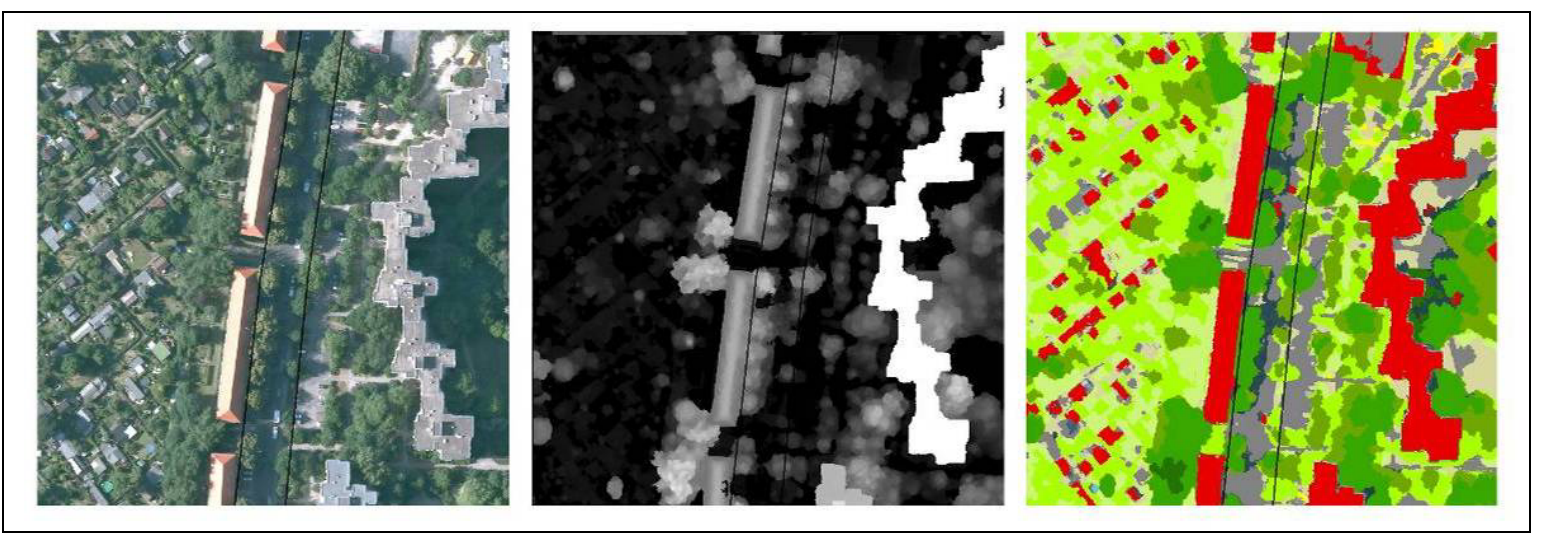

Figure 10: Classification result for a typical settlement with allotments.

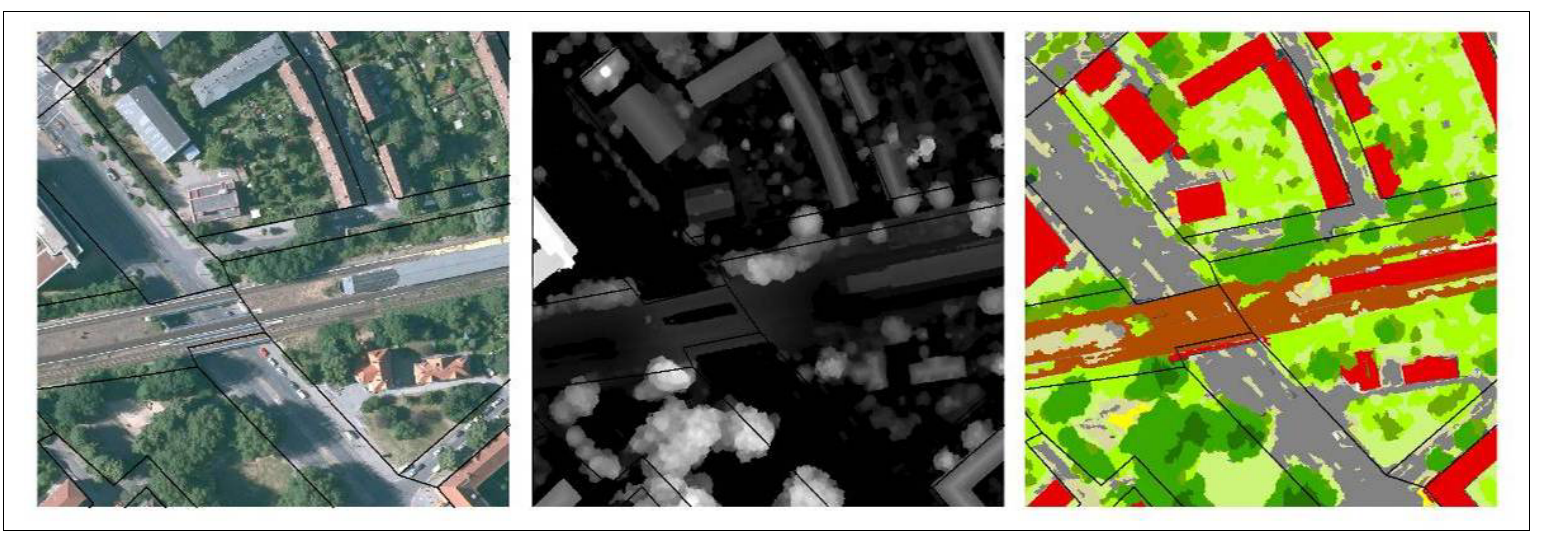

Figure 11: Classification result for a traffic area with railway tracks.

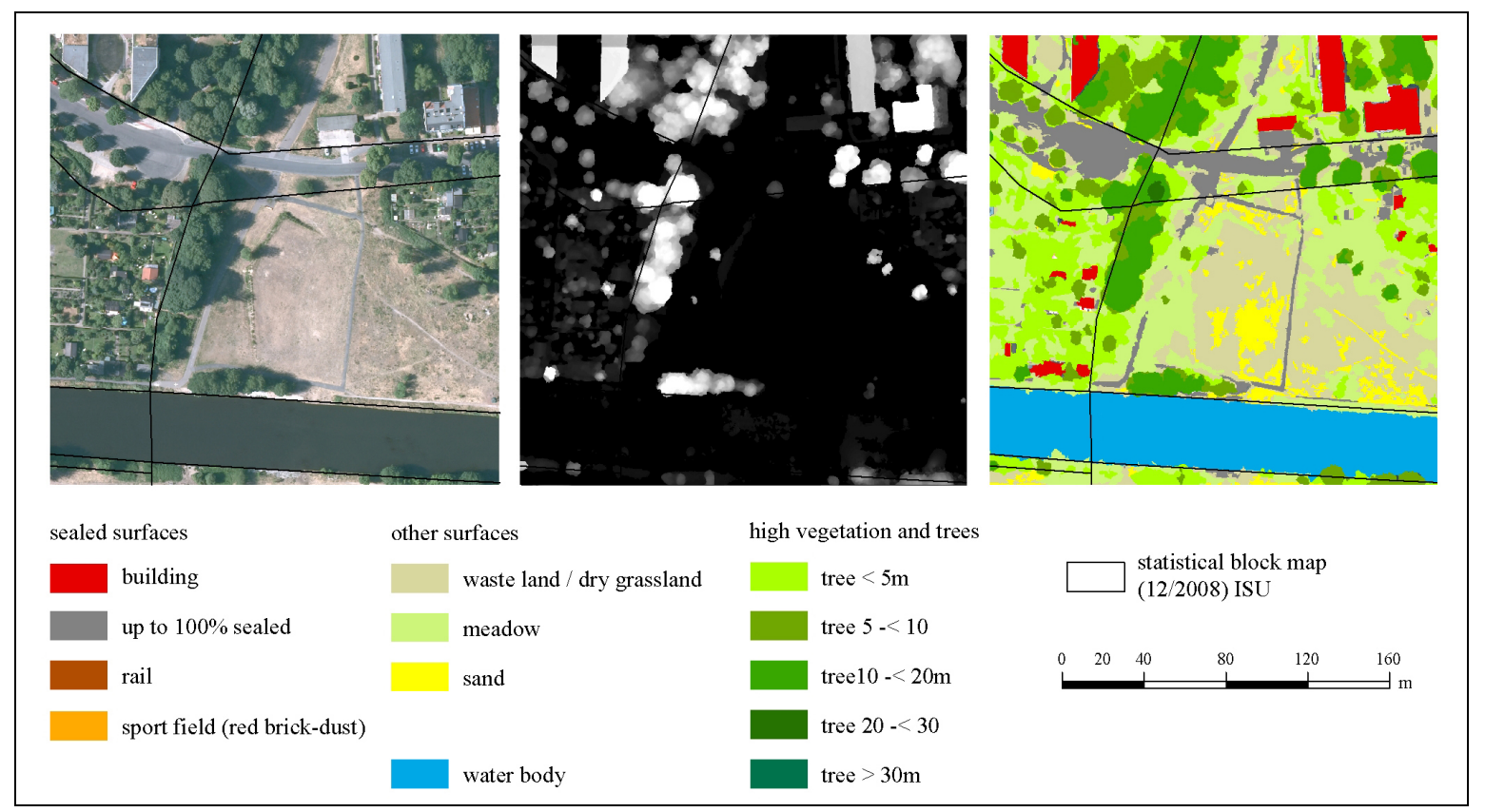

Figure 12: Classification result for the structure type open space, green and water body. 


\subsection{Accuracy assessment}

Only the results of first stage (coarse) classifications were investigated for its accuracy using random pixel samples in ERDAS Imagine software. These pixels as a component of a classified object were visually compared to UCX multispectral data. A total of 2500 samples were randomly selected. It has to be noticed, that due to the amount of the points this assessment is still ongoing. Comparing the first results to these of the previous studies it can be concluded, that very good results are to be expected. The Conditional Kappa statistic for the class buildings reaches 0,96 which is more than satisfactory. The explanation for this high accuracy is on the one hand the usage of ALK but on the other hand also its successful correction and the very accurate nDSM data. The results of the complete accuracy assessment will be presented on the conference.

\section{CONCLUSION AND OUTLOOK}

The study shows that airborne remote sensing imagery proves to be a valuable instrument for urban applications. A flight missions with high overlap $(80 \% / 60 \%)$ was used to produce a very detailed digital surface model and a true orthoimage mosaic both with $15 \mathrm{~cm} \mathrm{x} / \mathrm{y}$ resolution for Berlin. Additional vector data (ALK, ISU5) was introduced in the interpretation process. Segmentation and classification based on DSM, nDSM, TOM and the vector data already produced highly accurate classes with very high kappa coefficients in a two-step approach. The obtained blockwise statistics is significant, and even a subdivision into smaller units (e.g. buildings) appears to be reasonable. The study results are supportive not only for the ongoing $\mathrm{PhD}$ theses but also for future studies, e.g. in the fields of spatial analysis for energy and network providers or in the fields of urban ecology. The classification of shaded areas shows already on the basis of 8 bit data promising results, which will be improved in the near future due to the 16 bit data which is being processed now. For a full view into courtyards, data sets outside of the growing season are needed i.e. an aerial survey will be conducted in the winter months. The present images in $0.15 \mathrm{~m}$ and the height data set has numerous advantages compared to standard orthoimages, nevertheless also the constraints should be considered. One restriction is reflected in the large data volume, a stepwise procedure is necessary to get crucial solutions. With regard to the complexity of the study, it should be considered that it is still ongoing and does not claim to be complete. With additional multispectral images of previous and following years the change in the urban environment and related urban structure will be steadily monitored and analyzed. Furthermore we are working on a combination of different scales within one project, which allows a more meaningful analysis of semantic relationships.

\section{REFERENCES}

[1] Hirschmüller, H., "Stereo Vision in Structured Environment by Consistent Semi-Global Matching." In Proc. of the IEEE International Conference on Computer Vision and Pattern Recognition (CVPR 2006), New York, NY, USA. (2006).

[2] Maktav, D., Erbek, F. S. and Jürgens, C., "Remote Sensing of urban areas" International Journal of Remote Sensing, Vol. 26, No. 4, 655-659 (2005).

[3] Wurm, M., Taubenböck, H., Roth, A. and Dech, S., "Urban structuring using multisensoral remote sensing data by the example of German cities - Cologne and Dresden" In: Urban Remote Sensing Joint Event, Shanghai, China, (2009).

[4] Commission for Climatology and applications of Meteorology (WMO), "A bridged final report 8th session", WMO-No. 600 (1983).

[5] Trosset, A. M., Bucher, T. U. and Lehmann, F., "Adaption of building extraction rule sets derived from MFC3 and UltraCamD aerial image data sets", In Proc. of SPIE, Vol. 7478, (2009).

[6] Haag, L., Coenradie, B., Kleinschmit, B., Hostert, P., Damm, A., Goedecke, M. and Schneider, T., „Hybrides Kartierungsverfahren der Bodenversiegelung im urbanen Raum - das Ergebnis für Berlin. Bodenschutz" 3/08, 8288 (2008).

[7] Sukopp, H., Blume, H.-P., "Stadtökologie”, G. Fischer, Stuttgart, 127 pp. (1998).

[8] Braun, J., "Aspects on True-Orthophoto Production", Photogrammetrische Woche, Stuttgart, 205-214, (2003).

[9] Hirschmüller, H. and Bucher, T., "Evaluation of Digital Surface Models by Semi-Global Matching", DGPF Tagungsband 19/2010, Dreiländertagung OVG, DGPF and SGPF, 571-580 (2010). 
[10] Senate Department for Urban Development (SenStadt), "Flächentypen - Eine Beschreibung der im Informationssystem Stadt und Umwelt (ISU) der Senatsverwaltung für Stadtentwicklung erfassten und verwalteten Struktur- und Flächennutzungskategorien von Berlin“ (2001).

[11] Mayer, S., "Automatisierte Objekterkennung zur Interpretation hochauflösender Bilddaten in der Erdfernerkundung“, Doctoral thesis, Humboldt University, Berlin, Germany, (2004).

[12] Vanderhaegen, S. and F. Canters, "Developing urban metrics to describe the morphology of urban areas at block level", The International Archives of the Photogrammetry, Remote Sensing and Spatial Information Sciences, Vol. XXXVIII-4/C7 (2010).

[13] Baatz, M. and Schäpe, A., "Multiresolution Segmentation: an optimization approach for high quality multi-scale image segmentation", Proc. AGIT-Symposium, 12-23 (2000).

[14] Blaschke, T., “Objektextraktion und regelbasierte Klassifikation von Fernerkundungsdaten: Neue Möglichkeiten für GIS-Anwender und Planer“, Schrenk, M. (Ed.), CORP’ 2000. Computergestützte Raumplanung, Wien, Vol. 1, 153-162, (2000).

[15] Hirzinger, G.,Bodenmüller, T.,Hirschmüller, H., Liu, R.,Sepp, W., Suppa, M., Abmayr, T. and Strackenbrock, B., "Photo-realistic 3D modelling - From robotics perception towards cultural heritage. International Workshop on Recording, Modeling and Visualization of Cultural Heritage, Asona, Switzerland, May 22-27 (2005).

[16] Gruber, M., "UltraCamX, the new Digital Aerial Camera System by Microsoft Photogrammetry", Fritsch D. (Ed.) Photogrammetric Week'07, 137-145, (2007).

[17] http://www.dlr.de/os/desktopdefault.aspx/tabid-6416/10528 read-23334 (accessed 22nd July 2010)

[18] http://www.stadtentwicklung.berlin.de/umwelt/umweltatlas/ic607.htm (accessed 2nd Aug 2010)

[19] http://www.stadtentwicklung.berlin.de/umwelt/umweltatlas/ib102.htm (accessed 2nd Aug 2010) 